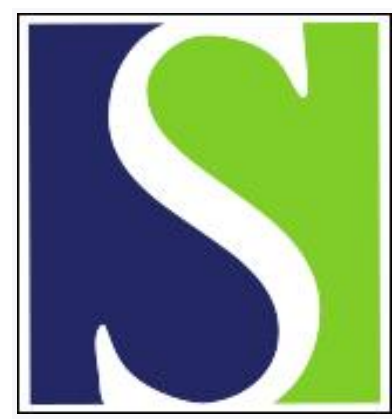

Scand J Work Environ Health 2012;38(2):171-181

https://doi.org/10.5271/sjweh.3197

Published online: 27 Sep 2011, Issue date: Mar 2012

Heart rate variability and endothelial function after sleep deprivation and recovery sleep among male shift and non-shift workers

by Wehrens SMT, Hampton SM, Skene DJ

Affiliation: Centre for Chronobiology, Faculty of Health and Medical Sciences, University of Surrey, Guildford, Surrey, GU2 7XH, UK. sophie.wehrens@surrey.ac.uk

Refers to the following texts of the Journal: 1999;25(2):85-99 2009;35(3):180-187

The following article refers to this text: 2014;40(6):621-630

Key terms: cardiovascular disease; endothelial function; heart rate; heart rate variability; parasympathetic activity; recovery sleep; restricted sleep; shift work; shift worker; sleep; sleep deprivation; sympathetic activity

This article in PubMed: www.ncbi.nlm.nih.gov/pubmed/21953310 


\title{
Heart rate variability and endothelial function after sleep deprivation and recovery sleep among male shift and non-shift workers
}

\author{
by Sophie MT Wehrens, PhD, ${ }^{1}$ Shelagh M Hampton, PhD, ${ }^{1}$ Debra J Skene, PhD ${ }^{1}$
}

\begin{abstract}
Wehrens SMT, Hampton SM, Skene DJ. Heart rate variability and endothelial function after sleep deprivation and recovery sleep among male shift and non-shift workers. Scand J Work Environ Health. 2012;38(2):171-181. doi:10.5271/sjweh.3197
\end{abstract}

Objectives Endothelial dysfunction and alterations in heart rate variability (HRV) as well as sleep deprivation and shift work have been associated with cardiovascular disease. The aim of this study was to compare HRV and endothelial function among shift and matched non-shift workers in response to total sleep deprivation and recovery sleep under identical laboratory settings.

Methods Eleven experienced male shift workers (shift work $\geq 5$ years) and 14 non-shift workers were matched for age, body mass index, and cholesterol. HRV parameters [eg, HR variance and low frequency/high frequency (LF/HF) ratio] were derived from 5-minute electrocardiogram bins at $0.25,4.25,11.5,12.5$, and 13.5 hours after habitual wake-up time and endothelial function was assessed by flow-mediated dilatation (FMD) using ultrasound at 0.75 and 10.75 hours after habitual wake-up time, following baseline sleep, total sleep deprivation, and recovery sleep (posture- and food-controlled throughout). Circadian phase was assessed before baseline sleep by salivary dim light melatonin onset.

Results There was no difference in circadian phase between shift and non-shift workers. HR variance was highest at 0.25 hours following total sleep deprivation and lowest after recovery sleep. A significantly higher $\mathrm{LF} / \mathrm{HF}$ ratio, significantly lower HR variance, and a trend for a lower \%FMD $(\mathrm{P}=0.08)$ were observed among shift compared to non-shift workers.

Conclusion Despite similar demographics, circadian phase, posture and food intake, differences in endothelial function and HRV were observed in the two groups, which may reflect higher sympathetic and/or lower parasympathetic activity, contributing to increased cardiovascular risk among the shift workers.

Key terms cardiovascular disease; parasympathetic activity; restricted sleep; shift work; sympathetic activity.

Epidemiological studies have shown that shift work is a risk factor for cardiovascular disease (CVD) (1-8). Assessing cardiovascular function by non-invasive methods is becoming increasingly popular and impaired endothelial function has been reported to be a good early predictor for CVD risk (9). Flow-mediated dilatation (FMD) using ultrasound (10) to assess endothelial function has been shown to be lower in populations with diabetes or coronary heart disease than healthy populations (11). Another indicator of cardiovascular function, heart rate variability (HRV) (12) is commonly assessed by time-domain measures derived from an electrocardiogram (ECG) [eg, the standard deviation (SD) of the normal-to-normal (NN) interval between QRS complexes (SDNN)]. In addition, the cardiac sympathetic/parasympathetic balance, is thought to be reflected in the low frequency/high frequency (LF/HF) ratio. A lower variance (eg, lower SDNN) in the ECG and a shift towards a higher LF/HF ratio (ie, higher sympathetic and/or lower parasympathetic activation) has been strongly associated with CVD and related conditions such as stress, metabolic syndrome, and diabetes (12-18).

Few studies have investigated endothelial function among shift workers. One field study has shown lower FMD among experienced shift workers (19). By contrast, HRV has been assessed more frequently in field studies of shift workers (20) with studies predominantly showing a shift in the sympathetic/parasympathetic balance. It is not known, however, what aspects of shift

1 Centre for Chronobiology, Faculty of Health and Medical Sciences, University of Surrey, Guildford, Surrey, UK.

Correspondence to: Sophie MT Wehrens, Centre for Chronobiology, Faculty of Health and Medical Sciences, University of Surrey, Guildford, Surrey, GU2 7XH, UK. [E-mail: sophie.wehrens@surrey.ac.uk or d.skene@surrey.ac.uk] 
work are causative. For example, it is still not clear how many years of shift work are needed before these changes become apparent. In addition, it is thought that the risk for CVD among shift workers may depend on the extent of circadian misalignment and sleep deprivation (21). Although it is thought that sleep deprivation and the risk for CVD are linked $(22,23)$, only a few studies have reported higher sympathetic activity and decreased vasodilatation during and after (partial) sleep deprivation (24-26).

Previous studies have often been confounded by differences in body posture, physical activity, stress levels, sleep opportunity, and circadian phase between conditions as well as a poor description of the shift work population and lack of an appropriate non-shift work control group. This has made it unclear whether the observations are due to confounding factors, chronic effects of shift work, or acute sleep deprivation per se. Moreover, to date, shift workers have not been compared directly to non-shift workers in the same study, endothelial function under these conditions has not been assessed by the gold standard ultrasound method, and HRV parameters in the time domain have not been reported widely. Finally, the effectiveness of interventions such as a recovery nap and recovery sleep to counteract the cardiovascular consequences of sleep deprivation after a night shift, have rarely been investigated.

The aim of this study was to investigate the effect of one night of total sleep deprivation, a recovery nap, and recovery sleep on FMD and HRV in controlled laboratory conditions and to assess the responses of experienced shift compared to non-shift workers in the same study. It was hypothesized that exposure to shift work would decrease the ability of the cardiovascular system to cope with sleep deprivation, as both shift work and sleep deprivation have been shown to have a negative impact on aspects of cardiovascular function.

\section{Methods}

The protocol has been described in detail elsewhere (27). A brief summary and details of procedures specific for this report (HRV and FMD) are presented.

\section{Procedures}

The University of Surrey Ethics Committee gave approval for all aspects of this study and the study was conducted in accordance with the Helsinki Declaration of 1975, as revised in 2000. All volunteer information was kept coded and held in strictest confidence. All participants gave written informed consent.

\section{Screening}

Pre-study screening and the laboratory part of the study took place in the clinical investigation unit at the University of Surrey. Participants were free of any medical conditions (eg, they were not hypertensive) and medication known to affect cardiovascular, metabolic, gastrointestinal, or immune function (including overthe-counter medication). Participants did not suffer from sleep, depression, or anxiety disorders as confirmed by the self-reported pre-study questionnaires and written confirmation from their general practitioner. All participants were currently non-smokers and did not consume $>15$ units of alcohol a week (recommended maximum intake in the UK is 21 units per week for men). A urine screen confirmed all subjects were negative for drugs of abuse at the time of recruitment and during the study.

\section{Participants}

Male shift and non-shift workers aged between 25-45 years were recruited. Shift workers were required to have a cumulative shift work history of $\geq 5$ years, working either permanent nights or rotating shifts with $\geq 3$ night shifts per month. Of the shift workers that participated in the study, 8 worked in the public and 3 in the private sector. One of the shift workers had finished shift work 18 months prior to the start of the study. Shift schedules were not controlled for before imposition of the regular sleep-wake cycle the week prior to the laboratory study (see below). The group of shift workers consisted of 3 night shift workers and 8 rotating shift workers [ $\mathrm{N}=3$ on a 2 -shift system (days and nights), $\mathrm{N}=2$ on a backward-rotating system and $\mathrm{N}=3$ on a forwardrotating system]. Demographics of the 25 participants (14 non-shift and 11 shift workers) are shown in table 1.

\section{Pre-laboratory session}

In order to ensure entrained circadian rhythms (especially important for the shift workers to avoid any acute effects of shift work-related circadian misalignment) and minimize sleep debt, volunteers were asked to keep a regular, self-selected sleep-wake cycle (28) with a nocturnal sleep duration of 7.5 or 8 hours, 8 days prior to the laboratory study. Participants thus scheduled either day work or days off prior to the study. This was confirmed with a timestamped voicemail, L-actiwatches (Cambridge Neurotechnology, Cambridge, UK), and sleep diaries (29). The participants' self-selected mean bed time, wake-up time, and sleep duration the week prior to the study were similar among shift workers (23:24, SD 00:36 hours; 07:12, SD 00:36 hours; and 07:54, SD 00:12 hours, respectively) and non-shift workers (23:18, SD 00:24 hours; 07:12, SD 00:30 hours; and 07:54, SD 00:12 hours, respectively). 
Table 1. Characteristics of non-shift and shift workers. [BMI=body mass index; HDL=high-density lipoprotein; SD=standard deviation].

\begin{tabular}{|c|c|c|c|c|c|c|}
\hline & \multicolumn{3}{|c|}{ Non-shift workers $(\mathrm{N}=14)$} & \multicolumn{3}{|c|}{ Shift workers $(N=11)$} \\
\hline & Mean & SD & Range & Mean & SD & Range \\
\hline Shift work (years) & 0.03 & 0.11 & $0-0.42$ & 8.7 & $5.2^{\mathrm{a}}$ & $5.0-18.5$ \\
\hline Time since last shift (months) & 60 & & & 1.6 & 5.4 & $0.0-18.0$ \\
\hline Age (years) & 32.5 & 6.2 & $25.0-42.0$ & 35.7 & 7.2 & $25.0-45.0$ \\
\hline Weight (kg) & 83.7 & 13.5 & $67.8-109.0$ & 91.6 & 10.3 & $82.0-117.0$ \\
\hline Height (m) & 1.77 & 0.06 & $1.66-1.85$ & 1.79 & 0.07 & $1.71-1.91$ \\
\hline $\mathrm{BMI}\left(\mathrm{kg} / \mathrm{m}^{2}\right)$ & 26.6 & 3.4 & $21.5-34.0$ & 28.7 & 3.8 & $23.0-35.7$ \\
\hline Waist circumference $(\mathrm{cm})$ & 91.2 & 9.4 & $76.0-107.0$ & 98.0 & 6.7 & $90.0-110.0$ \\
\hline Hip circumference $(\mathrm{cm})$ & 100 & 7.4 & $88.0-114.0$ & 105.6 & 6.2 & $95.0-114.0$ \\
\hline Waist-hip ratio & 0.91 & 0.04 & $0.84-0.96$ & 0.93 & 0.03 & $0.86-0.98$ \\
\hline Cholesterol (mmol/L) & 4.7 & 0.5 & $3.9-5.5$ & 4.9 & 1.1 & $3.0-6.5$ \\
\hline $\mathrm{HDL}(\mathrm{mmol} / \mathrm{L})$ & 1.3 & 0.2 & $0.8-1.6$ & 1.4 & 0.4 & $0.9-2.4$ \\
\hline Leptin $(\mathrm{ng} / \mathrm{mL})$ & 9.2 & 1.6 & $1.1-20.1$ & 10.5 & 1.3 & $5.6-17.4$ \\
\hline Caffeinated drinks (units/day) & 1.7 & 1.5 & $0-5-5.0$ & 3.0 & 2.3 & $0.0-7.5$ \\
\hline Currently smoke (units/day) & 0.0 & 0.0 & $0.0-0.0$ & 0.0 & 0.0 & $0.0-0.0$ \\
\hline Past smoked (units/day) & 3.6 & 5.8 & $0.0-15.5$ & 4.5 & 5.7 & $0.0-15$ \\
\hline Smoking duration (years) & 2.6 & 4.4 & $0.0-14.0$ & 4.4 & 6.3 & $0.0-20$ \\
\hline Time since last smoked (years) & 1.5 & 2.0 & $0.04-5.0$ & 3.2 & 4.5 & $0.02-11.0$ \\
\hline
\end{tabular}

a $\mathrm{P}<0.001$ compared to non-shift workers by independent two-tailed T-test.

Two days prior to the laboratory study, participants were asked to refrain from heavy exercise and substances that affect FMD and HRV such as alcohol and caffeine $(30,31)$ and food and drinks high in antioxidants (30) and nitrites/nitrates (31).

\section{Laboratory session}

Volunteers spent four days and nights in the clinical investigation unit (figure 1). After an adaptation night and baseline night (sleep equal to habitual sleep, 7.5 or 8 hours), subjects were required to stay awake for 30.5 hours until their 4-hour recovery nap, as a proxy for a night shift and subsequent sleep period. This was followed by recovery sleep at habitual bed time. During wake time, subjects were continually monitored by staff. Polysomnography was used to assess wakefulness during the sleep deprivation period post hoc. In order to control for inter-individual differences in circadian phase, all interventions and measurements were scheduled relative to each participant's self-selected wake-up time, which can be used as a proxy for circadian phase (32).

Body posture was controlled throughout the study. During assigned periods, subjects were asked to remain in a semi-recumbent position in bed. The semi-recumbent periods were the sleep and sleep deprivation periods and 4-hour periods each afternoon (ie, from 6.5-10.5 hours after wake time). In addition, every day $0.25,4.25,11.5$, 12.5 , and 13.5 hours after habitual wake-up time, the subjects were required to sit still for $\geq 15$ minutes for assessments. For $\geq 5$ minutes before and during each ultrasound recording, subjects were supine. Physical activity was further restricted during blood and saliva sampling (completed regularly for 5.75 hours from wake time and for 4 hours before bed time) as changes in body posture may influence saliva melatonin concentrations (33).

All subjects received the same standard meals throughout the study and were not allowed any food or drinks except for water between meals. The entire study was conducted in dim light $(<8$ lux in the direction of gaze) apart from darkness (0 lux) during the sleep periods. Light levels were regularly checked with a calibrated lux meter (Edmund Optics, York, UK). Temperature in the clinical investigation unit was set at $20^{\circ} \mathrm{C}$.

\section{Assessment of circadian phase}

Saliva was collected into scintillation vials (PerkinElmer, Beaconsfield, UK) every 30 minutes for 4 hours before the participants' self-selected bed time. Salivary melatonin concentrations were measured on the evening before baseline sleep according to an established protocol (34). The average limit of detection for the saliva melatonin assay was $1.5, \mathrm{SD} 1.2 \mathrm{pg} / \mathrm{ml}(\mathrm{N}=5)$. The inter-assay coefficients of variation were $15.9,13.0$, and $7.7 \%$ for quality control samples with $7.1(\mathrm{~N}=6), 19.5(\mathrm{~N}=6)$, and $44.1 \mathrm{pg} /$ $\mathrm{ml}(\mathrm{N}=5)$ melatonin, respectively. Dim light melatonin onset (DLMO) as a marker of circadian phase $(32,35$, 36) was determined for each subject as the time that the melatonin level exceeded the calculated threshold of $>2$ SD from the mean basal concentration (32). The DLMO could not be assessed for one shift worker due to elevated melatonin concentrations in the first saliva samples.

\section{Ultrasound recordings}

High-resolution recordings with an ultrasound machine (Acuson Aspen, Siemens, Bracknell, Berkshire, UK) were 


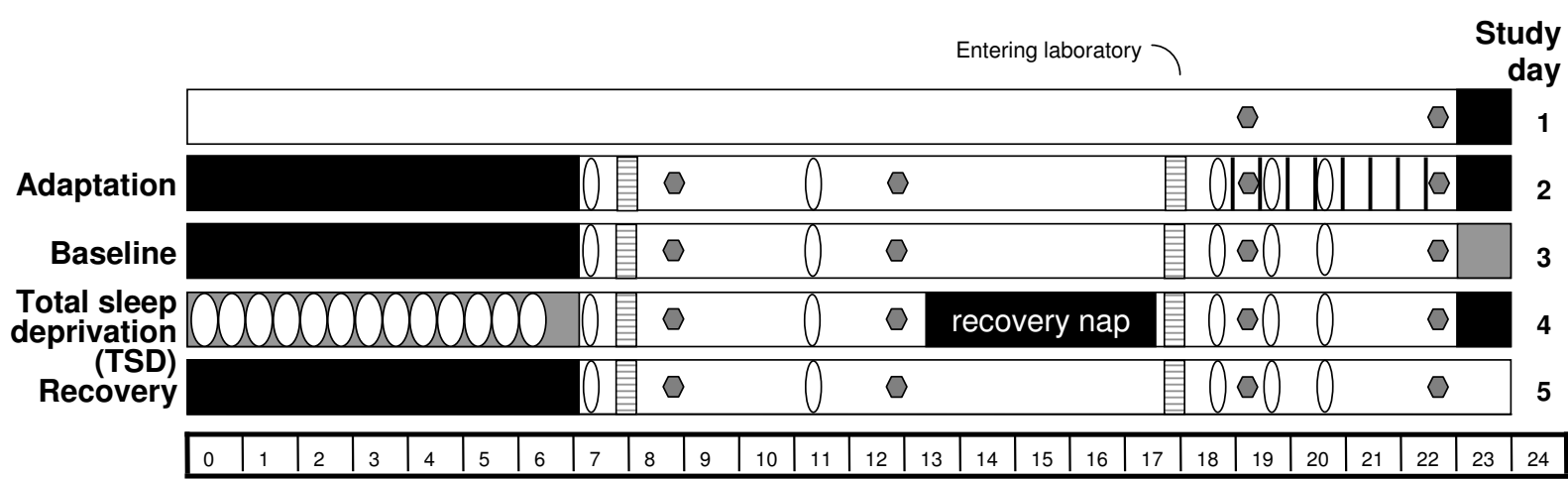

Clock time $(\mathrm{h})$

$\begin{array}{lllll}\text { 目 Ultrasound recordings } & 0 \text { HRV } & \square & \text { Wakefulness during TSD } & \text { All day }=\operatorname{dim} \text { light }<8 \text { lux } \\ \text { Saliva sampling } & 0 \text { Meal } & \text { Sleep (0 lux }) & \begin{array}{l}\text { Body posture controlled } \\ \text { throughout }\end{array}\end{array}$

Figure 1. Laboratory protocol. The diagram shows the 5 study days for a subject with sleep from 23:00-07:00 hours. All interventions (see key) were relative to each subject's self-selected wake time.

used to assess endothelial function by FMD at 0.75 and 10.75 hours after habitual wake-up time on each of the four study days. The method used in our laboratory (30, 31 ) was adapted from Donald et al $(37,38)$ and conducted in accordance with guidelines from the International Brachial Reactivity Task Force (39). Once the participant was supine and after a 5-minute rest, an 11-minute recording (Brachial Imager ${ }^{\mathrm{TM}}$, Iowa City, Iowa, USA) was started, capturing 220 ECG-triggered images of the brachial artery $5-10 \mathrm{~cm}$ above the antecubital fossa (during 1-minute rest, 5-minute occlusion, and 5-minute returning to rest). A second operator made changes in contrast and brightness during the recording if necessary.

The brachial artery diameter was determined within a region of interest for each of the 220 frames using edge detection software (Brachial Analyzer ${ }^{\mathrm{TM}}$ ). A fit of $>80 \%$ was aimed for and any falsely tracked edges were rejected. Maximum \%FMD was then calculated by the average of the 3 highest consecutive values for the diameter during and after cuff deflation as a percentage change from the average of 20 frames of resting diameters. The analysis was carried out by two experienced operators. Thus, this study used several standards to decrease variability and increase reproducibility such as brachial diameter calculation using 220 frames, ECG-triggered images, marking of the ultrasound probe location on the arm, and having the same operator set up and conduct the recording [as also suggested in methodological reviews by the Brachial Reactivity Task Force (39) and Stout (40)]. The intra-subject coefficients of variation averaged for all subjects for the morning and afternoon resting diameters were 2.9 and $3.3 \%$, respectively. These values were similar to an earlier reproducibility assessment by Walters et al (31). Some of the FMD data (7.3\%) were not usable because of missing data or low-quality recordings.

\section{HRV}

The ECG was derived from two positions: right midclavicular and around $6 \mathrm{~cm}$ under the left arm pit. The ECG electrodes were connected to a wireless polysomographic system (Siesta EEG/PSG recorder, Compumedics Ltd, Abbotsford, Victoria, Australia) and the ECG was continuously recorded at $256 \mathrm{~Hz}$ with ProFusion PSG 2 (version 2.1 build 134, Compumedics Ltd). The acquired data were converted into European data format and imported into Somnologica Studio (version 5.0.1 build 1662, Embla, supplied by Stowood Scientific Instruments Ltd, Oxford, UK) for further analysis. A value for each HRV parameter was generated every 5 minutes within a chosen analysis window. HRV was assessed at set periods. On each of the four study days, 5 -minute analysis windows were chosen $0.25,4.25$, $11.5,12.5$, and 13.5 hours after habitual wake-up time when the subjects were required to be semi-recumbent for other assessments. During the sleep deprivation night, data over 6.5 hours were split into $13 \times 30$-minute analysis periods (each period generating a value by averaging $6 \times 5$-minute analysis intervals) starting 17 hours after habitual wake-up time. The detection of QRS complexes was automatically done using Somnologica algorithm settings: (i) 5-minute analysis bins, (ii) data 
5 SD from the mean of the preceding 5 seconds of data were considered as potential R-tops, (iii) a minimum 5 consecutive R-tops were needed for a valid sequence, (iv) an output sampling rate of $1 \mathrm{~Hz}$ was used, and (v) minimum and maximum heart rate were set at 25 and 350 beats per minute, respectively, in compliance with the task force recommendations (12). Correct detection was manually confirmed and falsely detected QRS complexes (artifacts) were removed $(<1 \%$ of the analysis period for the daytime assessments and $<3 \%$ for the sleep deprivation night).

A HRV report based on the artifact-free data was automatically generated containing the following parameters: $\mathrm{NN}$ interval (interval between adjacent QRS complexes), square root of the mean of the sum of the squares of differences between NN intervals (RMSSD), SD of all $\mathrm{NN}$ intervals $(\mathrm{SDNN}) /$ mean of the SD of all NN intervals for all 5-minute segments in the recording (SDNN index), SD of differences between NN intervals (SDSD), percentage of NN intervals $>50 \mathrm{~ms}$ (pNN50), very low frequency power (VLF: 0.003-0.04 Hz), low frequency power (LF: $0.04-0.15 \mathrm{~Hz}$ ), high frequency power (HF: $0.15-0.4 \mathrm{~Hz}$ ), total power (TP), $\mathrm{LF}_{\text {norm }}[\mathrm{LF} /(\mathrm{LF}+\mathrm{HF})]$, $\mathrm{HF}_{\text {norm }}[\mathrm{HF} /(\mathrm{LF}+\mathrm{HF})]$, and LF/HF. All 13 analysis bins for the sleep deprivation night were complete apart from one non-shift worker whose NN-interval data were excluded as an outlier ( $>2.3 \mathrm{SD}$ from the mean). For the 5-minute analysis bins, some data were missing and some bins were considered outliers according to the same criteria (total missing values comprised $<7 \%$ of the dataset).

\section{Statistical analysis}

The average DLMO among shift and non-shift workers was compared using an independent two-tailed Student's T-test. The HRV assessments during the sleep deprivation night were analyzed using twofactor ANOVA [factors "time" (13 levels) and "group" (shift and non-shift workers)] (SPSS 16, SPSS Inc, IBM company, Chicago, Illinois, USA). If applicable, P-values were Greenhouse-Geisser-adjusted when Mauchly's test for sphericity was significant.

A mixed-model regression analysis was conducted in SAS 9.1 (TS level 1M3) (SAS Institute Inc, Cary, NC, USA) for the daytime FMD and HRV assessments. The repeated-measures model determined main effects (group, day, and time) and all interactions between them, using the Prasad-Rao-Jeske-Kackar-Harville fixedeffects method, with a variance components covariance structure and the Kenward-Roger method for calculating degrees of freedom. The data after adaptation sleep were excluded from the analyses. To locate post hoc differences between days and time points, least squares means were calculated and t-type confidence limits were constructed for each of the least squares means.

\section{Results}

\section{Assessment of circadian phase}

Figure 2 shows the average melatonin profiles for shift and non-shift workers on the evening before baseline sleep. The time of onset of the melatonin rhythm was not significantly different among shift workers [DLMO 21.1, standard error of the mean (SEM) 0.2 hours], compared to non-shift workers (DLMO 21.2, SEM 0.2 hours). The time interval (phase angle) between melatonin onset and self-selected bed time was also not significantly different between the shift (2.2, SEM 0.1 hours) and non-shift workers (2.1, SEM 0.2 hours).

\section{Percent flow-mediated dilatation}

There was no significant effect of day or time or day $\times$ time interaction on the resting brachial diameter. There was a significant effect of group $\left(\mathrm{F}_{1,127}=10.2, \mathrm{P}<0.01\right)$, however, with a significantly higher resting diameter among shift compared to non-shift workers. Body weight as a random effect was significantly associated with resting diameter $(\mathrm{P}<0.001)$ and the observed effect of group on resting diameter became statistically non-significant when body weight was used as a random effect.

The \%FMD among both shift and non-shift workers in the morning and afternoon is shown in figure 3. There were no significant effects of day, time, group, or interactions on \%FMD. A trend for a group effect, however, was observed $\left(\mathrm{F}_{1,127}=3.0, \mathrm{P}=0.08\right)$ with a lower $\% \mathrm{FMD}$ among shift compared to non-shift workers.

\section{Heart rate variability during total sleep deprivation}

The SDNN index and LF/HF ratio during total sleep deprivation are shown in figure 4. Average values for the other HRV parameters across the total sleep deprivation night are shown in table 2 . There was a significant effect of time of day on SDNN $\left(\mathrm{F}_{4.6,106}=8.9, \mathrm{P}<0.001\right)$, VLF $\left(\mathrm{F}_{7.1,164}=11.1, \mathrm{P}<0.001\right), \operatorname{LF}\left(\mathrm{F}_{5.6,129}=2.7, \mathrm{P}<0.05\right)$, and TP $\left(\mathrm{F}_{6.6,151}=11.5, \mathrm{P}<0.001\right)$ with the values increasing throughout the night of total sleep deprivation. There were no significant effects of group, time, or interactions on any of the other HRV parameters.

\section{Heart rate variability across the study days}

The SDNN and LF/HF ratio after baseline sleep, during and after sleep deprivation, and after recovery sleep are shown in figure 5. Although for completeness the data during sleep deprivation are shown again in figure 5, the statistical analysis mentioned hereafter only includes the 5 $\times 5$-minute daytime points after baseline sleep, total sleep deprivation, and recovery sleep. There was a significant 


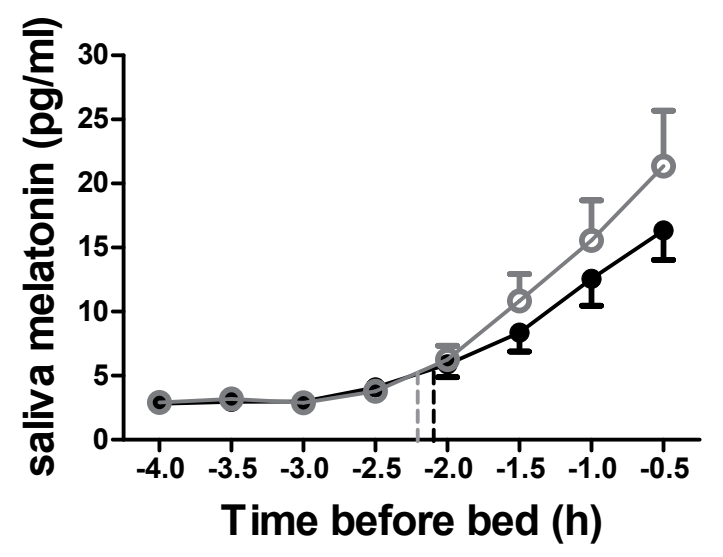

Figure 2. Saliva melatonin concentrations (mean and standard error of the mean) among non-shift $(\bullet)$ and shift workers (०) 4-0.5 hours prior to habitual bed time. Dashed lines indicate dim-light melatonin onset (DLM0) with respect to bed time (phase angles) among shift (grey, $\mathrm{N}=10$ ) and non-shift workers (black, $\mathrm{N}=14$ ).

effect of group $\left(\mathrm{F}_{1,325}=12.9, \mathrm{P}<0.001\right)$ and a significant day $\times$ time interaction $\left(\mathrm{F}_{8,325}=2.0, \mathrm{P}<0.05\right)$ on $\mathrm{SDNN}$. SDNN was significantly lower among shift compared to non-shift workers $(\mathrm{P}<0.001)$. The most consistent observation emerging from the day $\times$ time interaction was that SDNN following a night of sleep deprivation $(0.25$ hours after habitual wake up time) was significantly higher than at all other time points $(\mathrm{P}<0.001$ for all points apart from 0.25 hours after waking following baseline sleep which was $\mathrm{P}<0.01$ ) (figure $5 \mathrm{a}$ ).

There was also a significant effect of group $\left(\mathrm{F}_{1,311}=5.5\right.$, $\mathrm{P}<0.05)$ and time of day $\left(\mathrm{F}_{4,311}=8.6, \mathrm{P}<0.001\right)$ on $\mathrm{LF} / \mathrm{HF}$, which was significantly higher among shift than non-shift workers $(\mathrm{P}<0.05)$. LF/HF was significantly higher 13.5 hours after waking than at 4.25 hours after waking and significantly higher at $13.5,12.5$, and 4.25 hours than at 11.5 and 0.25 hours after waking (figure $5 b$ ).

Most of the other HRV parameters also showed significant effects of group, day and time. The pNN50 showed the same effects as SDNN. The RMSSD, SDSD, and NN were significantly lower after recovery sleep compared to baseline sleep and total sleep deprivation. In addition, RMSSD and SDSD were significantly lower among shift than non-shift workers. NN was higher at 0.25 hours after waking than at all other time points and higher at 11.5 hours than at $4.25,12.5$, and 13.5 hours after waking. LF norm had the same significant effects as LF/HF and estimates for the effects were in the same direction, whereas HF norm showed a time $\times$ group interaction. The VLF, LF, HF, and TP were lower after recovery sleep compared to baseline sleep and total sleep deprivation. These parameters also showed a significant effect of time of day with higher values at
Table 2. Heart rate variability (HRV) parameters of non-shift and shift workers during the sleep deprivation night. [SEM=standard error of the mean; HF=high frequency power $(0.15-0.4 \mathrm{~Hz})$; $\mathrm{HF}_{\text {norm }}=$ normalized $\mathrm{HF}(\mathrm{HF} /[\mathrm{LF}+\mathrm{HF}])$ in normalized units (nu); $L F=$ low frequency power $(0.04-0.15 \mathrm{~Hz})$; $L_{\text {norm }}=$ normalized $L F$ $(\mathrm{LF} /[\mathrm{LF}+\mathrm{HF}])$ in nu; NN-interval=normal-to-normal interval (intervals between adjacent [QRS] complexes); pNN50=percentage of NN intervals $>50 \mathrm{~ms}$; RMSSD=square root of the mean of the sum of the squares of differences between NN intervals; SDNN index=mean of the standard deviations (SD) of all NN intervals for all 5-minute segments in the recording; $S D S D=S D$ of differences between NN intervals; TP=total power; VLF=very low frequency power $(0.003-0.04 \mathrm{~Hz})$.]

\begin{tabular}{|c|c|c|c|c|}
\hline & \multicolumn{2}{|c|}{$\begin{array}{l}\text { Non-shift workers } \\
\qquad(\mathrm{N}=14)\end{array}$} & \multicolumn{2}{|c|}{$\begin{array}{l}\text { Shift workers } \\
\qquad(N=11)\end{array}$} \\
\hline & Mean & SEM & Mean & SEM \\
\hline \multicolumn{5}{|l|}{ Time domain } \\
\hline NN interval (ms) & 917 & 26 & 937 & 24 \\
\hline pNN50 (\%) & 22 & 4 & 18 & 3 \\
\hline SDNN index (ms) & 88 & 6 & 87 & 7 \\
\hline $\operatorname{SDSD}(\mathrm{ms})$ & 33 & 4 & 31 & 3 \\
\hline RMSSD (ms) & 47 & 5 & 42 & 4 \\
\hline \multicolumn{5}{|l|}{ Frequency domain } \\
\hline $\mathrm{TP}\left(\mathrm{ms}^{2}\right)$ & 20664 & 1455 & 21225 & 1896 \\
\hline $\operatorname{VLF}\left(\mathrm{ms}^{2}\right)$ & 13216 & 1307 & 13563 & 1501 \\
\hline $\mathrm{LF}\left(\mathrm{ms}^{2}\right)$ & 6014 & 477 & 6386 & 644 \\
\hline $\mathrm{HF}\left(\mathrm{ms}^{2}\right)$ & 1335 & 112 & 1179 & 132 \\
\hline LF/HF & 5.3 & 0.6 & 6.3 & 0.6 \\
\hline LF norm (nu) & 0.8 & 0.0 & 0.9 & 0.0 \\
\hline HF norm (nu) & 0.19 & 0.02 & 0.15 & 0.02 \\
\hline
\end{tabular}

0.25 hours after waking than at all other time points and higher values at 4.25 and 11.5 hours than at 12.5 and 13.5 hours in most cases.

\section{Discussion}

The present study investigated the effects of sleep deprivation and recovery sleep on FMD and HRV in controlled laboratory conditions. For the first time, experienced shift workers were compared directly to non-shift workers matched for age, body mass index, and cholesterol levels in an identical laboratory setting with strict control of body posture, physical activity, meals, circadian phase, and ambient lighting.

Although the shift schedule was not controlled for prior to the laboratory study, participants were asked to keep a regular sleep-wake cycle ( 7.5 or 8 hours nocturnal sleep) for 8 days before the laboratory session. No significant difference between the DLMO among shift and non-shift workers confirmed that the participants' circadian phase was controlled in the study. The shift workers were normally entrained to the light-dark cycle showing comparable phase angles between DLMO and bedtime compared to the non-shift workers and to previous reports among healthy young volunteers (32). All 
A

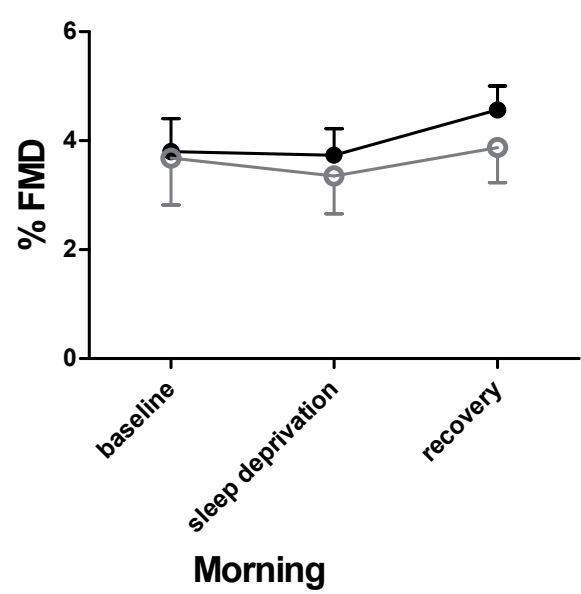

A

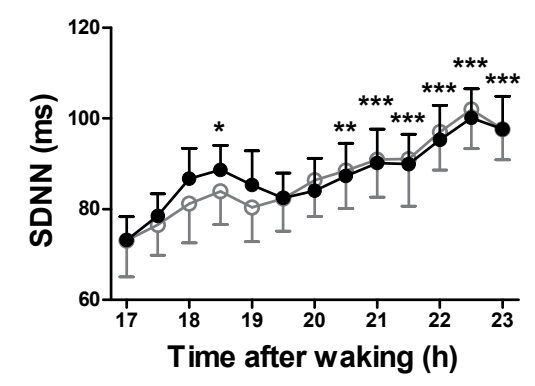

B

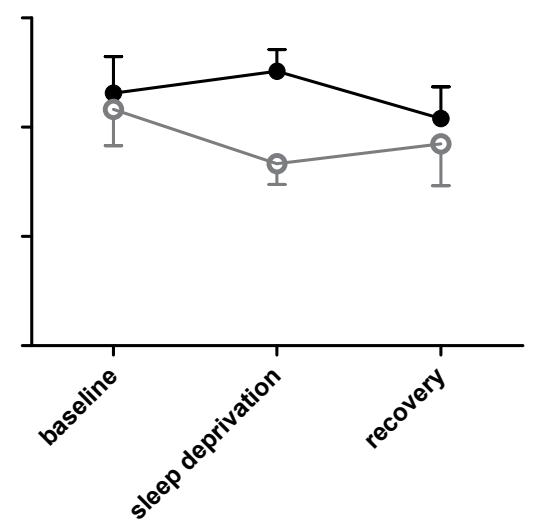

Afternoon

B

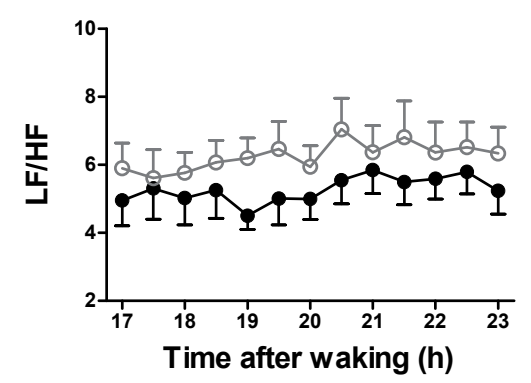

Figure 3. (A) Percent flowmediated dilatation (\%FMD) (mean and standard error of the mean) in the morning ( 0.75 hours after habitual wake time) and (B) the afternoon (10.75 hours after habitual wake time), following baseline sleep, total sleep deprivation, and recovery sleep among non-shift $(\bullet)$ and shift workers (०). $\mathrm{N}=14$ for non-shift workers and $\mathrm{N}=11$ for shift workers.
Figure 4. (A) The standard deviation of the normal-to normal interval between QRS complexes (SDNN) and (B) low frequency/ high frequency (LF/HF) ratio (mean and standard error of the mean) among non-shift $(\bullet)$ and shift workers (o) during total sleep deprivation (from 17-23 hours after habitual wake time). ${ }^{*} P<0.05,{ }^{* *} P<0.01$ and *** $P<0.001$ compared to 17 hours after waking. $\mathrm{N}=14$ for non-shift workers and $\mathrm{N}=11$ for shift workers. the FMD and HRV measurements were thus taken at a similar circadian phase for all subjects and therefore any observed group differences are unlikely to be due to differences in circadian timing.

The results showed a trend towards a lower FMD among the shift workers. This finding is in agreement with a field study by Amir and colleagues (19) showing that baseline FMD was significantly lower among physicians who had worked shifts for $>3$ years compared to those with a shorter shift work history. These findings indicate a higher sympathetic tone among shift workers as reduced vasodilatation can result from acute sympathetic stimulation (41-43). Shift and non-shift workers also showed significant differences in HRV parameters: SDNN, RMSSD, and SDSD were significantly lower and LF/HF was significantly higher among the shift workers. Lower SDNN, RMSSD, and SDSD reflect a lower variance and higher LF/HF indicates higher sympathetic and/or lower parasympathetic activity, both factors being associated with a higher risk of CVD $(13,15,17)$. The current findings thus support the idea of increased cardiovascular risk among shift workers. Previous studies have shown that normalized LF power or LF/HF is higher on night duty than when off duty and asleep at night (44-49) and is higher on days including a night shift compared to days including a morning shift (50). Normalized LF power or LF/HF has also been shown to be higher among shift compared to non-shift workers when both groups work day shifts $(51,52)$ or are asleep at home $(53)$. One study of shift workers found lower SDNN and RMSDD during a night shift compared to sleep at night (45). By contrast, some studies have not been able to show this association between higher LF/HF and lower variability and shift work (54-56). The current study confirmed that these predominant observations of higher LF/HF or normalized LF power and lower variance are also detectable among shift workers studied in a controlled laboratory setting. In addition to differences between the groups, the HRV data also showed significant effects of time of day. Changes within a day and circadian rhythms with peaks in the early morning have been previously reported in LF, HF (57), and SDNN (57-59), but not in other HRV parameters such as $\operatorname{LF} / \operatorname{HF}(57,58)$. 
A baseline

sleep deprivation

recovery

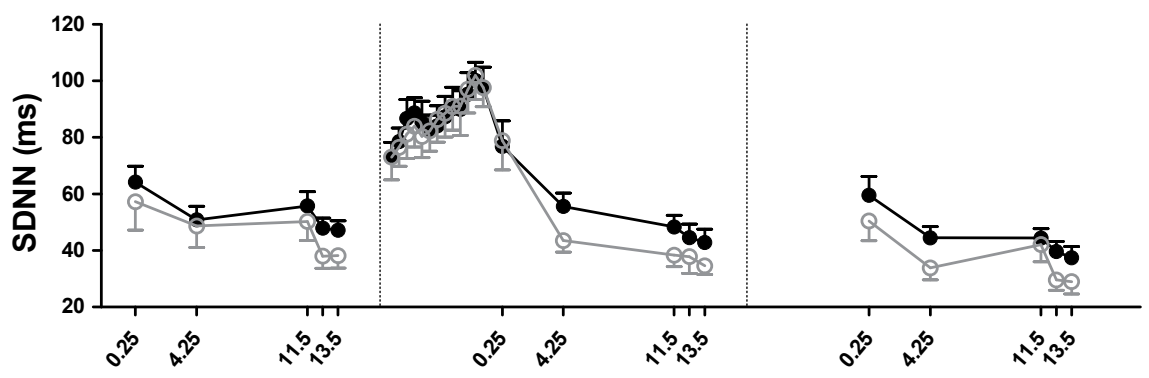

B

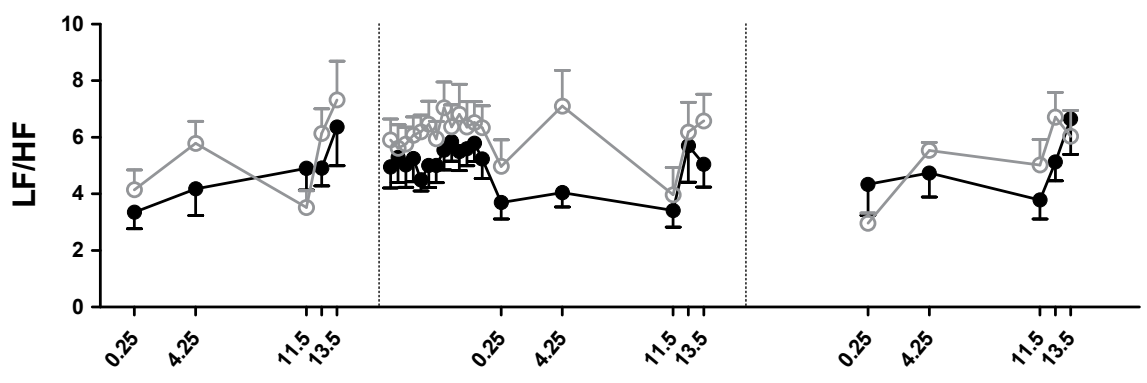

Time after waking (h)
Figure 5.(A) The standard deviation of the normal-to normal interval between QRS complexes (SDNN) and (B) low frequency/high frequency (LF/HF) ratio (mean and standard error of the mean) among non-shift $(\bullet)$ and shift workers (०) 0.25 , $4.25,11.5,12.5$, and 13.5 hours after habitual wake time following baseline sleep, total sleep deprivation and recovery sleep. $\mathrm{N}=14$ for non-shift workers and $\mathrm{N}=11$ for shift workers.
This study aimed not only to compare shift and nonshift workers but also to investigate the effects of sleep deprivation and recovery sleep on FMD and HRV. In contrast to previous studies $(19,24)$, the current study did not find a significant decrease in FMD after total sleep deprivation. Sauvet et al (24) observed a decreased cutaneous vascular conductance after 29 hours of total sleep deprivation (by both endothelium-dependent and endothelium-independent vasodilatation) but not after 35 hours when assessed by endothelium-dependent vasodilatation. In our study, FMD was measured after 24.75 hours of total sleep deprivation and after 34.75 hours following total sleep deprivation plus a 4-hour nap. This may be one reason for the differences in the findings between the two studies. Moreover, discrepancies between the studies could be due to other methodological differences (eg, circadian phase and physical activity was controlled for in this study). In addition, Sauvet et al (24) used a method that is substantially different from the ultrasound technique employed in our study as they did not assess flow-mediated dilatation in the main blood vessels but rather cutaneous microcirculation using the technique of iontophoresis-induced dilatation. The HRV parameters, however, showed significant differences across study days. The significantly higher SDNN observed following a night of sleep deprivation is in agreement with a study from Viola et al (58). A few studies have reported significantly higher LF/HF and/or normalized LF power
(24-26), a longer NN interval (60), and a lower NN50 (46) during and after total and partial sleep deprivation. Other studies, however, have found no difference in HRV parameters in these conditions $(61,62)$. The current study showed lower RMSSD and SDSD after recovery sleep indicating lower variance and a decrease in $\mathrm{NN}$-interval throughout the study. These changes after recovery sleep parallel our postprandial metabolism data obtained in the same protocol (27), where significant changes in basal and postprandial metabolic and insulin responses were also observed after recovery sleep. Another study also found significant increases in intercellular adhesion molecule-1, interleukin-6, and norepinephrine after recovery sleep but not after total sleep deprivation, which may play a role in alterations in the sympathetic/parasympathetic balance and vascular tone (24). A longer period of recovery sleep would therefore be needed to investigate further the time course of these observed changes following recovery sleep.

Differences in cardiovascular function between the shift and non-shift workers were observed despite the fact that screening parameters (eg, cholesterol and body mass index) were not significantly different between the two groups. FMD and HRV measurements may thus be more sensitive in detecting early cardiovascular alterations. A previous study has also proposed that attenuated FMD may predict cardiovascular dysfunction (63). Both the FMD and HRV data suggest that shift workers exert 
higher levels of sympathetic activity/lower parasympathetic activity on the cardiovascular system contributing to their increased cardiovascular risk. To further investigate this mechanism, it would be of interest to assess other markers of sympathetic activity such as the catecholamines in these groups. The reliability of these assessments, however, has been questioned and thus they cannot replace spectral analysis of HRV as the method of choice to assess autonomic function.

It is not possible to extract a single cause for the observed differences between the shift and non-shift workers. The long-term effects of shift work may be a plausible explanation as all shift workers in the current study had worked shifts for $\geq 5$ years. In addition, repeated sleep deprivation and/or circadian misalignment of rhythms in HRV and endothelial function with the imposed lifestyle (eg, sleep-wake cycle and physical activity) among unadapted shift workers could contribute to the effect of shift work duration on HRV.

In conclusion, group differences between shift and non-shift workers were observed. Shift workers showed a trend for a lower FMD and a significantly higher LF/ HF ratio, suggesting higher sympathetic and/or lower parasympathetic activity. In addition, they displayed significantly lower HR variance (lower SDNN, RMSSD, and SDSD). Whether these HRV changes reflect the increased CVD risk reported in this group requires further study.

\section{Acknowledgements}

We would like to thank researchers and staff at the University of Surrey for their help, especially Prof Christopher Fry and Dr Rita Jabr for discussions about the ECG and Mr Peter Williams for statistical advice. In addition we would like to thank Mr Jussi Virkkala (Finnish Institute of Occupational Health \& Tampere University Hospital) for advice on HRV analysis.

This work was supported by an EU Marie Curie Research Training Network grant (CT-2004-512362), $6^{\text {th }}$ Framework project EUCLOCK (018741) and Stockgrand Ltd, University of Surrey, Guildford, UK.

\section{References}

1. Boggild H, Knutsson A. Shift work, risk factors and cardiovascular disease. Scand J Work Environ Health. 1999;25:85-99.

2. De Bacquer D, Van Risseghem M, Clays E, Kittel F, De Backer G, Braeckman L. Rotating shift work and the metabolic syndrome: a prospective study. Int J Epidemiol. 2009 Jun;38(3):848-54.
3. Ellingsen T, Bener A, Gehani AA. Study of shift work and risk of coronary events. J R Soc Promot Health. 2007;127:265-7. http://dx.doi.org/10.1177/1466424007083702.

4. Esquirol Y, Bongard V, Mabile L, Jonnier B, Soulat J-M, Perret B. Shift Work and Metabolic Syndrome: Respective Impacts of Job Strain, Physical Activity, and Dietary Rhythms. Chronobiol Int. 2009;26:544-59. http://dx.doi. org/10.1080/07420520902821176.

5. Karlsson B, Knutsson A, Lindahl B. Is there an association between shift work and having a metabolic syndrome? Results from a population based study of 27,485 people. Occup Environ Med. 2001;58:747-52. http://dx.doi.org/10.1136/ oem.58.11.747.

6. Karlsson BH, Knutsson AK, Lindahl BO, Alfredsson LS Metabolic disturbances in male workers with rotating threeshift work. Results of the WOLF study. Int Arch Occup Environ Health. 2003; 76:424-30. http://dx.doi.org/10.1007/ s00420-003-0440-y.

7. Sookoian S, Gemma C, Fernandez Gianotti T, Burgueno A, Alvarez A, Gonzalez CD, et al. Effects of rotating shift work on biomarkers of metabolic syndrome and inflammation. J Intern Med. 2007;261:285-92.

8. Kawachi I, Colditz GA, Stampfer MJ, Willett WC, Manson JE, Speizer FE, et al. Prospective study of shift work and risk of coronary heart disease in women. Circulation. 1995;92:3178-82.

9. Vita JA, Keaney JF, Jr. Endothelial function: a barometer for cardiovascular risk? Circulation. 2002;106:640-2. http:// dx.doi.org/10.1161/01.CIR.0000028581.07992.56.

10. Celermajer DS, Sorensen KE, Gooch VM, Spiegelhalter DJ, Miller OI, Sullivan ID, et al. Non-invasive detection of endothelial dysfunction in children and adults at risk of atherosclerosis. Lancet. 1992;340:1111-5.

11. Bots ML, Westerink J, Rabelink TJ, de Koning EJ. Assessment of flow-mediated vasodilatation (FMD) of the brachial artery: effects of technical aspects of the FMD measurement on the FMD response. Eur Heart J. 2005;26:363-8.

12. Task Force of the European Society of Cardiology and the North American Society of Pacing and Electrophysiology. Heart rate variability. Standards of measurement, physiological interpretation, and clinical use. Eur Heart J. 1996;17:354-81.

13. Buccelletti E, Gilardi E, Scaini E, Galiuto L, Persiani R, Biondi $A$, et al. Heart rate variability and myocardial infarction: systematic literature review and metanalysis. Eur Rev Med Pharmacol Sci. 2009;13:299-307.

14. Koskinen T, Kahonen M, Jula A, Mattsson N, Laitinen T, Keltikangas-Jarvinen L, et al. Metabolic syndrome and shortterm heart rate variability in young adults. The cardiovascular risk in young Finns study. Diabet Med. 2009;26:354-61. http://dx.doi.org/10.1111/j.1464-5491.2009.02686.x.

15. Rabbone I, Bobbio A, Rabbia F, Bertello MC, Ignaccoldo MG, Saglio E, et al. Early cardiovascular autonomic dysfunction, beta cell function and insulin resistance in obese adolescents. Acta Biomed. 2009;80:29-35.

16. Manfrini O, Pizzi C, Viecca M, Bugiardini R. Abnormalities 
of cardiac autonomic nervous activity correlate with expansive coronary artery remodeling. Atherosclerosis. 2008;197:183-9. http://dx.doi.org/10.1016/j.atherosclerosis.2007.03.013.

17. Perciaccante A, Fiorentini A, Paris A, Serra P, Tubani L. Circadian rhythm of the autonomic nervous system in insulin resistant subjects with normoglycemia, impaired fasting glycemia, impaired glucose tolerance, type 2 diabetes mellitus. BMC Cardiovasc Disord. 2006;6:19. http://dx.doi. org/10.1186/1471-2261-6-19.

18. Thayer JF, Yamamoto SS, Brosschot JF. The relationship of autonomic imbalance, heart rate variability and cardiovascular disease risk factors. Int J Cardiol. 2010;141:122-31. http:// dx.doi.org/10.1016/j.ijcard.2009.09.543.

19. Amir O, Alroy S, Schliamser JE, Asmir I, Shiran A, Flugelman MY, et al. Brachial artery endothelial function in residents and fellows working night shifts. Am J Cardiol. 2004;93:947-9. http://dx.doi.org/10.1016/j.amjcard.2003.12.032.

20. Togo F, Takahashi M. Heart rate variability in occupational health -a systematic review. Ind Health. 2009;47:589-602. http://dx.doi.org/10.2486/indhealth.47.589.

21. Knutsson A, Boggild H. Shiftwork and cardiovascular disease: review of disease mechanisms. Rev Environ Health. 2000;15:359-72. http://dx.doi.org/10.1515/ REVEH.2000.15.4.359.

22. Gangwisch JE, Heymsfield SB, Boden-Albala B, Buijs RM, Kreier F, Pickering TG, et al. Short sleep duration as a risk factor for hypertension: analyses of the first National Health and Nutrition Examination Survey. Hypertension. 2006;47:833-9. http://dx.doi.org/10.1161/01.HYP.0000217362.34748.e0.

23. Taheri S, Lin L, Austin D, Young T, Mignot E. Short sleep duration is associated with reduced leptin, elevated ghrelin, and increased body mass index. PLoS Med. 2004;1:e62. http:// dx.doi.org/10.1371/journal.pmed.0010062.

24. Sauvet F, Leftheriotis G, Gomez-Merino D, Langrume C, Drogou C, Van Beers P, et al. Effect of acute sleep deprivation on vascular function in healthy subjects. J Appl Physiol. 2009;108:68-75. http://dx.doi.org/10.1152/ japplphysiol.00851.2009.

25. Spiegel K, Leproult R, Van Cauter E. Impact of sleep debt on metabolic and endocrine function. The Lancet. 1999;354:14359. http://dx.doi.org/10.1016/S0140-6736(99)01376-8.

26. Zhong X, Hilton HJ, Gates GJ, Jelic S, Stern Y, Bartels MN, et al. Increased sympathetic and decreased parasympathetic cardiovascular modulation in normal humans with acute sleep deprivation. J Appl Physiol. 2005;98:2024-32. http://dx.doi. org/10.1152/japplphysiol.00620.2004

27. Wehrens SM, Hampton SM, Finn RE, Skene DJ. Effect of total sleep deprivation on postprandial metabolic and insulin responses in shift workers and non-shift workers. J Endocrinol. 2010;206:205-15. http://dx.doi.org/10.1677/JOE-10-0077.

28. Revell VL, Kim H, Tseng CY, Crowley SJ, Eastman CI. Circadian phase determined from melatonin profiles is reproducible after $1 \mathrm{wk}$ in subjects who sleep later on weekends. J Pineal Res. 2005;39:195-200. http://dx.doi. org/10.1111/j.1600-079X.2005.00236.x.
29. Lockley SW, Skene DJ, Arendt J. Comparison between subjective and actigraphic measurement of sleep and sleep rhythms. J Sleep Res. 1999;8:175-83. http://dx.doi. org/10.1046/j.1365-2869.1999.00155.x.

30. Hampton SM, Isherwood C, Kirkpatrick VJ, Lynne-Smith AC, Griffin BA. The influence of alcohol consumed with a meal on endothelial function in healthy individuals. J Hum Nutr Diet. 2010;23:120-5. http://dx.doi.org/10.1111/j.1365277X.2009.01021.x.

31. Walters JF, Hampton SM, Deanfield JE, Donald AE, Skene DJ, Ferns GA. Circadian variation in endothelial function is attenuated in postmenopausal women. Maturitas. 2006;54:294 303. http://dx.doi.org/10.1016/j.maturitas.2005.12.005.

32. Burgess HJ, Savic N, Sletten T, Roach G, Gilbert SS, Dawson D. The relationship between the dim light melatonin onset and sleep on a regular schedule in young healthy adults. Behav Sleep Med. 2003;1:102-14. http://dx.doi.org/10.1207/ S15402010BSM0102_3.

33. Deacon S, Arendt J. Posture influences melatonin concentrations in plasma and saliva in humans. Neurosci Lett. 1994;167:191-4. http://dx.doi.org/10.1016/03043940(94)91059-6.

34. English J, Middleton BA, Arendt J, Wirz-Justice A. Rapid direct measurement of melatonin in saliva using an iodinated tracer and solid phase second antibody. Ann Clin Biochem. 1993;30(Pt 4):415-6.

35. Arendt J. Importance and relevance of melatonin to human biological rhythms. J Neuroendocrinol. 2003;15:427-31. http://dx.doi.org/10.1046/j.1365-2826.2003.00987.x.

36. Klerman EB, Gershengorn HB, Duffy JF, Kronauer RE. Comparisons of the variability of three markers of the human circadian pacemaker. J Biol Rhythms. 2002;17:181-93. http:// dx.doi.org/10.1177/074873002129002474.

37. Donald AE, Charakida M, Cole TJ, Friberg P, Chowienczyk PJ, Millasseau SC, et al. Non-invasive assessment of endothelial function: which technique? J Am Coll Cardiol. 2006;48: 1846-50. http://dx.doi.org/10.1016/j.jacc.2006.07.039.

38. Charakida M, Donald AE, Terese M, Leary S, Halcox JP, Ness A, et al. Endothelial dysfunction in childhood infection. Circulation. 2005;111:1660-5. http://dx.doi.org/10.1161/01. CIR.0000160365.18879.1C.

39. Corretti MC, Anderson TJ, Benjamin EJ, Celermajer D, Charbonneau F, Creager MA, et al. Guidelines for the ultrasound assessment of endothelial-dependent flowmediated vasodilation of the brachial artery - A report of the International Brachial Artery Reactivity Task Force. J Am Coll Cardiol. 2002;39:257-65. http://dx.doi.org/10.1016/S07351097(01)01746-6.

40. Stout M. Flow-mediated dilatation: a review of techniques and applications. Echocardiography. 2009;26:832-41. http:// dx.doi.org/10.1111/j.1540-8175.2009.00927.x.

41. Dyson KS, Shoemaker JK, Hughson RL. Effect of acute sympathetic nervous system activation on flow-mediated dilation of brachial artery. Am J Physiol Heart Circ Physiol. 2006; 290:H1446-53. http://dx.doi.org/10.1152/ ajpheart.00771.2005. 
42. Iversen S, Iversen L, Saper C. Chapter 49 The Autonomic Nervous System and the Hypothalamus. In: Kandel ER, Schwartz JH, Jesell TM. Principles of Neural Science. New York: McGraw-Hill, Health Professions Division, 2000, p960-81.

43. Hijmering ML, Stroes ES, Olijhoek J, Hutten BA, Blankestijn PJ, Rabelink TJ. Sympathetic activation markedly reduces endothelium-dependent, flow-mediated vasodilation. J Am Coll Cardiol. 2002;39:683-8. http://dx.doi.org/10.1016/ S0735-1097(01)01786-7.

44. Rauchenzauner M, Ernst F, Hintringer F, Ulmer H, Ebenbichler $\mathrm{CF}$, Kasseroler MT, et al. Arrhythmias and increased neuroendocrine stress response during physicians' night shifts: a randomized cross-over trial. Eur Heart J. 2009;30:2606-13. http://dx.doi.org/10.1093/eurheartj/ehp268.

45. Su TC, Lin LY, Baker D, Schnall PL, Chen MF, Hwang WC, et al. Elevated blood pressure, decreased heart rate variability and incomplete blood pressure recovery after a 12-hour night shift work. J Occup Health. 2008;50:380-6. http://dx.doi. org/10.1539/joh.L7056.

46. Ewing DJ, Neilson JM, Shapiro CM, Stewart JA, Reid W. Twenty four hour heart rate variability: effects of posture, sleep, and time of day in healthy controls and comparison with bedside tests of autonomic function in diabetic patients. Br Heart J. 1991;65:239-44. http://dx.doi.org/10.1136/ hrt.65.5.239.

47. Furlan R, Barbic F, Piazza S, Tinelli M, Seghizzi P, Malliani A Modifications of Cardiac Autonomic Profile Associated With a Shift Schedule of Work. Circulation. 2000;102:1912-6.

48. Ito H, Nozaki M, Maruyama T, Kaji Y, Tsuda Y. Shift work modifies the circadian patterns of heart rate variability in nurses. Int J Cardiol. 2001;79:231-6. http://dx.doi. org/10.1016/S0167-5273(01)00439-9.

49. Mitani S, Fujita M, Shirakawa T. Circadian variation of cardiac autonomic nervous profile is affected in Japanese ambulance men with a working system of 24-h shifts. Int Arch Occup Environ Health. 2006;79:27-32. http://dx.doi.org/10.1007/ s00420-005-0026-y.

50. Van Amelsvoort LG, Schouten EG, Maan AC, Swenne CA, Kok FJ. 24-Hour heart rate variability in shift workers: impact of shift schedule. J Occup Health. 2001;43:33-8.

51. Ishii N, Dakeishi M, Sasaki M, Iwata T, Murata K. Cardiac autonomic imbalance in female nurses with shift work. Auton Neurosci. 2005;122:94-9. http://dx.doi.org/10.1016/j. autneu.2005.08.010.

52. Ishii N, Iwata T, Dakeishi M, Murata K. Effects of shift work on autonomic and neuromotor functions in female nurses. J Occup Health. 2004;46:352-8. http://dx.doi.org/10.1539/ joh.46.352.

53. Chung MH, Kuo TB, Hsu N, Chu H, Chou KR, Yang CC. Sleep and autonomic nervous system changes - enhanced cardiac sympathetic modulations during sleep in permanent night shift nurses. Scand J Work Environ Health. 2009;35:180-7.
54. van Amelsvoort LG, Schouten EG, Maan AC, Swenne CA, Kok FJ. Changes in frequency of premature complexes and heart rate variability related to shift work. Occup Environ Med. 2001; 58:678-81. http://dx.doi.org/10.1136/oem.58.10.678.

55. Freitas J, Lago P, Puig J, Carvalho MJ, Costa O, de Freitas AF. Circadian heart rate variability rhythm in shift workers. J Electrocardiol. 1997;30:39-44. http://dx.doi.org/10.1016/ S0022-0736(97)80033-7.

56. Murata K, Yano E, Hashimoto H, Karita K, Dakeishi M. Effects of shift work on QTc interval and blood pressure in relation to heart rate variability. Int Arch Occup Environ Health. 2005;78: 287-92. http://dx.doi.org/10.1007/s00420004-0592-4.

57. Vandewalle G, Middleton B, Rajaratnam SM, Stone BM, Thorleifsdottir B, Arendt J, et al. Robust circadian rhythm in heart rate and its variability: influence of exogenous melatonin and photoperiod. J Sleep Res. 2007;16:148-55. http://dx.doi. org/10.1111/j.1365-2869.2007.00581.x.

58. Viola AU, Simon C, Ehrhart J, Geny B, Piquard F, Muzet A, et al. Sleep processes exert a predominant influence on the 24-h profile of heart rate variability. J Biol Rhythms. 2002;17: 539-47. http://dx.doi.org/10.1177/0748730402238236.

59. Bonnemeier H, Richardt G, Potratz J, Wiegand UK, Brandes A, Kluge N, et al. Circadian profile of cardiac autonomic nervous modulation in healthy subjects: differing effects of aging and gender on heart rate variability. J Cardiovasc Electrophysiol. 2003;14:791-9. http://dx.doi.org/10.1046/ j.1540-8167.2003.03078.x.

60. Pagani M, Pizzinelli P, Traon AP, Ferreri C, Beltrami S, Bareille MP, et al. Hemodynamic, autonomic and baroreflex changes after one night sleep deprivation in healthy volunteers. Auton Neurosci. 2009;145:76-80. http://dx.doi. org/10.1016/j.autneu.2008.10.009.

61. Gould KS, Hirvonen K, Koefoed VF, Roed BK, Sallinen M, Holm A, et al. Effects of 60 hours of total sleep deprivation on two methods of high-speed ship navigation. Ergonomics. 2009;52: 1469-86. http://dx.doi.org/10.1080/00140130903272611.

62. Muenter NK, Watenpaugh DE, Wasmund WL, Wasmund SL, Maxwell SA, Smith ML. Effect of sleep restriction on orthostatic cardiovascular control in humans. J Appl Physiol. 2000;88:966-72.

63. Shechter M, Issachar A, Marai I, Koren-Morag N, Freinark D, Shahar Y, et al. Long-term association of brachial artery flow-mediated vasodilation and cardiovascular events in middle-aged subjects with no apparent heart disease. Int J Cardiol. 2009;134:52-8. http://dx.doi.org/10.1016/j. ijcard.2008.01.021.

Received for publication: 26 May 2011 